\title{
Photon spectrum produced by the late decay of a cosmic neutrino background
}

\author{
Eduard Massó \\ Grup de Física Teòrica and Institut de Física d'Altes Energies, Universitat Autònoma de Barcelona, \\ 08193 Bellaterra, Catalonia, Spain \\ Ramon Toldrà \\ NASA/Fermilab Astrophysics Group, Fermi National Accelerator Laboratory, Box 500, Batavia, Illinois 60510-0500
}

(Received 1 April 1999; published 8 September 1999)

\begin{abstract}
We obtain the photon spectrum induced by a cosmic background of unstable neutrinos. We study the spectrum in a variety of cosmological scenarios and also we allow for the neutrinos having a momentum distribution (only a critical matter-dominated universe and neutrinos at rest have been considered until now). Our results can be helpful when extracting bounds on neutrino electric and magnetic moments from cosmic photon background observations. [S0556-2821(99)00118-6]

PACS number(s): 98.80.-k, 14.60.St
\end{abstract}

\section{INTRODUCTION}

Recent indications for neutrino masses [1] have strengthened the interest in physical effects linked to massive neutrinos. In this paper we shall be concerned with one of these effects, namely, with the fact that massive neutrinos can have nonzero electromagnetic form factors and can be unstable due to radiative decay processes.

Specifically, we shall be interested in the contribution of decays $\nu_{i} \rightarrow \nu_{j} \gamma$, when coming from a cosmological $\nu_{i}$ density, to the cosmic photon background. A previous study of such phenomena was performed by Ressell and Turner [2] (see also [3]). These authors considered the photon background at different photon energies and constrained the radiative decay of a cosmic density of massive neutrinos. Recently, Biller et al. [4] have improved substantially the constraints in the infrared region by making use of the effect of $\gamma-\gamma$ interactions giving electron-positron pairs, where one $\gamma$ is a background infrared photon, and the other is a $\mathrm{TeV} \gamma$ coming from an active galaxy. We may expect further improvements in the future, along the lines used in [4] or perhaps with some new ideas and/or observations.

Constraints on the neutrino lifetime lead to limits on the magnetic and electric transition moments, $\mu_{i j}$ and $\epsilon_{i j}$, of neutrinos. The neutrino lifetime $\tau$ is related to these parameters,

$$
\frac{B}{\tau}=\frac{\left|\mu_{i j}\right|^{2}+\left|\epsilon_{i j}\right|^{2}}{8 \pi}\left(\frac{\Delta m^{2}}{m_{i}}\right)^{3}
$$

where $\Delta m^{2}=m_{i}^{2}-m_{j}^{2}$, and $B$ is the branching ratio $B R\left(\nu_{i}\right.$ $\left.\rightarrow \nu_{j} \gamma\right)$.

All the studies performed until now make the simplifying assumptions that the decays are produced (1) in a critical matter-dominated universe and (2) by neutrinos at rest. Although this might be enough for the results obtained until now, in the future it may be interesting to study the contribution to the photon background with more generality. In a more general framework we can be able to see how the limits on the neutrino lifetime depend on different assumptions, and we can extract more precise and reliable bounds. Also, in the eventual case of a positive signal that may come from decays of relic neutrinos, the general study of the consequences of the decay that we perform in the present paper would become quite necessary. Apart from the abovementioned references, other papers treating unstable neutrinos in a cosmological context are listed in [6].

Nonvanishing values of $\mu_{i j}$ and/or $\epsilon_{i j}$ lead to other potential effects besides the ones considered in this paper. For example, plasmon decay into neutrinos in stellar media is constrained by arguments of stellar energy loss. This leads, in general, to limits that are quite stringent [5]. We would like to point out that when neutrinos are close to a degeneration in mass, the limits obtained using Eq. (1) may be relevant since in this case $\Delta m^{2} / m_{i} \ll 1$. In any case, the spirit of the present paper is not phenomenological. Rather, as we said, we would like to generalize previous studies on the subject.

The organization of the paper is as follows. In Sec. II we examine the contribution to the photon background of the decay $\nu_{i} \rightarrow \nu_{j} \gamma$ in different cosmological scenarios, keeping the assumption that the neutrinos $\nu_{i}$ decay at rest. In Sec. III we drop this assumption and study the decays of neutrinos with a momentum distribution. We devote Sec. IV to the conclusions. Some technical details are developed in two appendixes.

\section{DECAYING NEUTRINOS AT REST}

In this section we shall calculate the photon spectrum produced by the decay of a cosmic background of neutrinos at rest. We shall consider the two-body decay of a neutrino with mass $m_{i}$ into a photon and a neutrino with mass $m_{j}$, with $m_{i}>m_{j}: \nu_{i} \rightarrow \nu_{j}+\gamma$. The subscripts $i, j=1,2,3$ stand for any neutrino mass eigenstate, which is a linear combination of the three weak eigenstates $\nu_{e}, \nu_{\mu}, \nu_{\tau}$. When a neutrino decays at rest the photon energy is given by

$$
\epsilon_{0}=\frac{\Delta m^{2}}{2 m_{i}}
$$


with $\Delta m^{2} \equiv m_{i}^{2}-m_{j}^{2}$. The cosmic expansion redshifts the photon energy. A photon that at present has energy $E$ was produced at a redshift $z_{0}$ given by

$$
1+z_{0}=\frac{\epsilon_{0}}{E}
$$

Let $F_{E}$ be the present energy flux of photons with energy $E$ produced by neutrino decay. The flux per unit energy and solid angle is given by

$$
\frac{d^{2} F_{E}}{d E d \Omega}=E \frac{d^{2} F_{n}}{d E d \Omega},
$$

where $F_{n}$ is the particle flux at present. It is related to the particle flux at emission time by the relation

$$
\frac{d^{2} F_{n}}{d \Omega}=\frac{1}{\left(1+z_{0}\right)^{3}} \frac{d^{2} F_{n}}{d \Omega}\left(t\left(z_{0}\right)\right)=\frac{1}{4 \pi} \frac{1}{\left(1+z_{0}\right)^{3}} \delta n_{\gamma}\left(t\left(z_{0}\right)\right),
$$

where we have included the factor of dilution $\left(1+z_{0}\right)^{-3}$ produced by the expansion of the universe. The photon density emitted at $z_{0}$ is given by the usual decay law

$$
\delta n_{\gamma}\left(t\left(z_{0}\right)\right)=B \frac{\delta t}{\tau} n_{\nu_{i}}\left(t\left(z_{0}\right)\right),
$$

where $\tau$ is the neutrino lifetime and $B$ is the branching ratio for the radiative decay. For a fixed emission time $\delta t$ $=H^{-1}\left(z_{0}\right) d E / E$, where $H(z)$ is the Hubble expansion rate at time $t(z)$. Writing everything together we obtain

$$
\frac{d^{2} F_{E}}{d E d \Omega}=\frac{1}{4 \pi} \frac{n_{v_{i}}\left(t\left(z_{0}\right)\right)}{\left(1+z_{0}\right)^{3}} \frac{B}{\tau H\left(z_{0}\right)} .
$$

Choosing a time $t_{p} \ll \tau$, otherwise arbitrary, and calling the expansion age of the universe $t_{0}$, we can write

$$
\begin{aligned}
\frac{n_{\nu_{i}}\left(t\left(z_{0}\right)\right)}{\left(1+z_{0}\right)^{3}} & =\frac{n_{v_{i}}\left(t_{p}\right)}{\left[1+z\left(t_{p}\right)\right]^{3}} \exp -\frac{t\left(z_{0}\right)-t_{p}}{\tau} \\
& \equiv \tilde{n}_{\nu_{i}}\left(t_{0}\right) \exp -\frac{t\left(z_{0}\right)-t_{p}}{\tau},
\end{aligned}
$$

where $\tilde{n}_{v_{i}}\left(t_{0}\right)$ would be the present number density of neutrinos if they did not decay. Taking $t_{p}=0$ and substituting Eq. (8) into Eq. (7), we finally obtain

$$
\frac{d^{2} F_{E}}{d E d \Omega}=\frac{B}{4 \pi} \frac{\tilde{n}_{\nu_{i}}\left(t_{0}\right)}{\tau H\left(z_{0}\right)} \exp -\frac{t\left(z_{0}\right)}{\tau},
$$

for any $E<\epsilon_{0}$. For $E>\epsilon_{0}$ the photon flux vanishes because photons cannot be produced with energy larger than $\epsilon_{0}$ by neutrinos decaying at rest. From now on we shall set $B=1$. The expansion time $t(z)$ is given in terms of $H(z)$ by the following integral:

$$
t(z)=\int_{0}^{t(z)} d t=\int_{z}^{\infty} \frac{d z^{\prime}}{1+z^{\prime}} \frac{1}{H\left(z^{\prime}\right)} .
$$

Equation (9) is our final expression for the photon energy flux per unit energy and solid angle produced by the decay of a cosmic background of neutrinos with negligible velocities. It holds for any isotropic and homogeneous universe and for any equation of state for the cosmic fluid.

Once the expansion rate $H(z)$ is given, one can calculate $t(z)$ with Eq. (10) and then calculate $H\left(z_{0}\right)$ and $t\left(z_{0}\right)$ using Eq. (3). Plugging the result into Eq. (9) one obtains the photon spectrum as a function of the photon energy $E$. As examples we shall consider three different cosmological scenarios: a power-law expansion and two observationally more motivated scenarios, an open universe filled only with matter and a flat universe with matter and a nonzero cosmological constant $\Lambda$.

First, let us take a power-law expansion. The Hubble expansion rate and the expansion time are given, respectively, by

$$
\begin{aligned}
H(z) & =H_{0}(1+z)^{n}, \\
H_{0} t(z) & =\frac{1}{n} \frac{1}{(1+z)^{n}} .
\end{aligned}
$$

with $n \geqslant 0$ and $H_{0}$ being the Hubble constant [7]. Particular examples include a critical $\Lambda$-dominated universe $(n=0)$, an empty open universe $(n=1)$, a flat matter-dominated universe $(n=3 / 2)$, and a flat radiation-dominated universe ( $n$ $=2$ ). For a critical matter-dominated universe we obtain

$$
\frac{d^{2} F_{E}}{d E d \Omega}=\frac{1}{4 \pi} \frac{\tilde{n}_{\nu_{i}}\left(t_{0}\right)}{\tau H_{0}}\left(\frac{E}{\epsilon_{0}}\right)^{3 / 2} \exp -\frac{2}{3} \frac{1}{\tau H_{0}}\left(\frac{E}{\epsilon_{0}}\right)^{3 / 2} .
$$

The only case studied in the literature is a critical matterdominated universe $\left(\Omega_{0}=8 \pi G \rho_{m} / 3 H_{0}^{2}=1\right.$, where $\rho_{m}$ is the present matter density) with $m_{j}=0[2-4]$. In this case and when $\tau \gg H_{0}^{-1}$ we obtain

$$
\frac{d^{2} F_{E}}{d E d \Omega}=\frac{1}{4 \pi} \frac{\tilde{n}_{\nu_{i}}\left(t_{0}\right)}{\tau H_{0}}\left(\frac{E}{m_{i} / 2}\right)^{3 / 2}
$$

in agreement with previous results.

For an open universe with matter density in critical density units $\Omega_{0}<1$, one has

$$
\begin{aligned}
H(z)= & H_{0} \sqrt{\Omega_{k}(1+z)^{2}+\Omega_{0}(1+z)^{3}}, \\
H_{0} t(z)= & \frac{1}{\Omega_{k}} \frac{\sqrt{\Omega_{k}+\Omega_{0}(1+z)}}{1+z} \\
& -\frac{\Omega_{0}}{\Omega_{k}^{3 / 2}} \ln \frac{\sqrt{\Omega_{k}}+\sqrt{\Omega_{k}+\Omega_{0}(1+z)}}{\sqrt{\Omega_{0}(1+z)}},
\end{aligned}
$$

where $\Omega_{k} \equiv 1-\Omega_{0}$. 


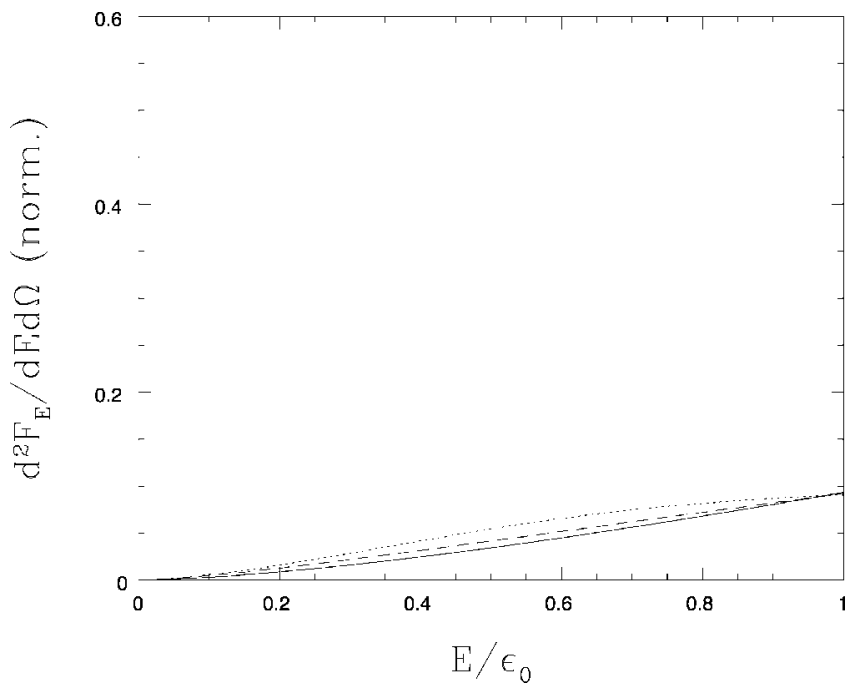

FIG. 1. We plot the normalized spectrum $\left(d^{2} F_{E} /\right.$ $d E d \Omega) /\left(\tilde{n}_{\nu_{i}}\left(t_{0}\right) / 4 \pi\right)$ versus $E / \epsilon_{0}$ when $\tau H_{0}=10$. The solid line represents the cosmological model $\Omega_{0}=1$ and $\Omega_{\Lambda}=0$. The dashed line is the model $\Omega_{0}=0.3$ and $\Omega_{\Lambda}=0$. The dotted line is $\Omega_{0}=0.3$ and $\Omega_{\Lambda}=0.7$.

The last example that we solve is a flat universe filled with matter and a nonvanishing cosmological constant $\Lambda$. The contents in matter is given by $\Omega_{0}$ and the contribution of the cosmological constant is $\Omega_{\Lambda} \equiv \Lambda / 3 H_{0}^{2}=1-\Omega_{0}$. The expansion rate and the expansion time at redshift $z$ are given, respectively, by

$$
\begin{aligned}
H(z) & =H_{0} \sqrt{\Omega_{\Lambda}+\Omega_{0}(1+z)^{3}}, \\
H_{0} t(z) & =\frac{2}{3 \sqrt{\Omega_{\Lambda}}} \ln \frac{\sqrt{\Omega_{\Lambda}}+\sqrt{\Omega_{\Lambda}+\Omega_{0}(1+z)^{3}}}{\sqrt{\Omega_{0}(1+z)^{3}}} .
\end{aligned}
$$

We plot in Figs. 1, 2, and 3 the photon spectrum (9) in the three cases listed above, for three different values of $\tau$,

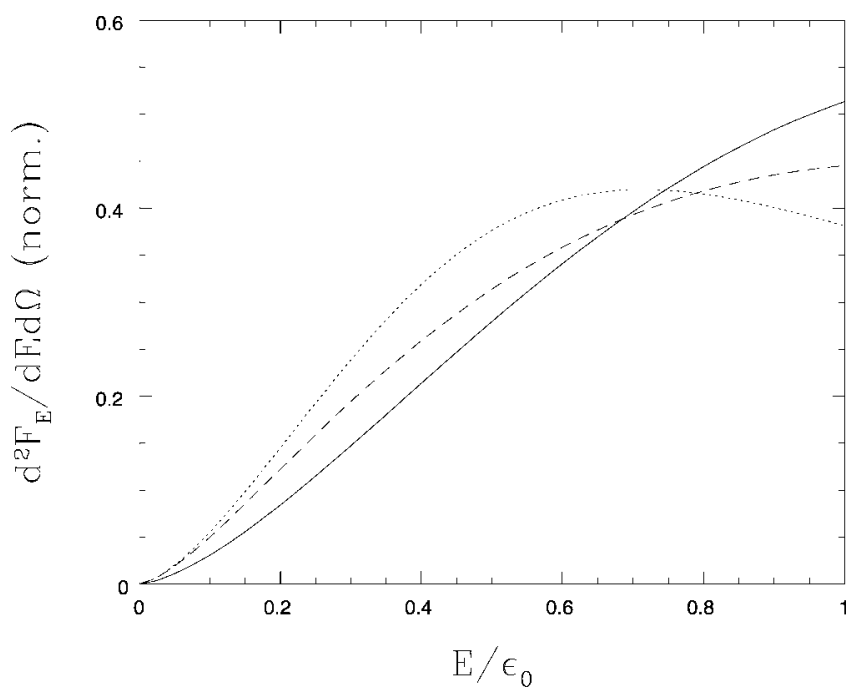

FIG. 2. Same as Fig. 1 but with $\tau H_{0}=1$.

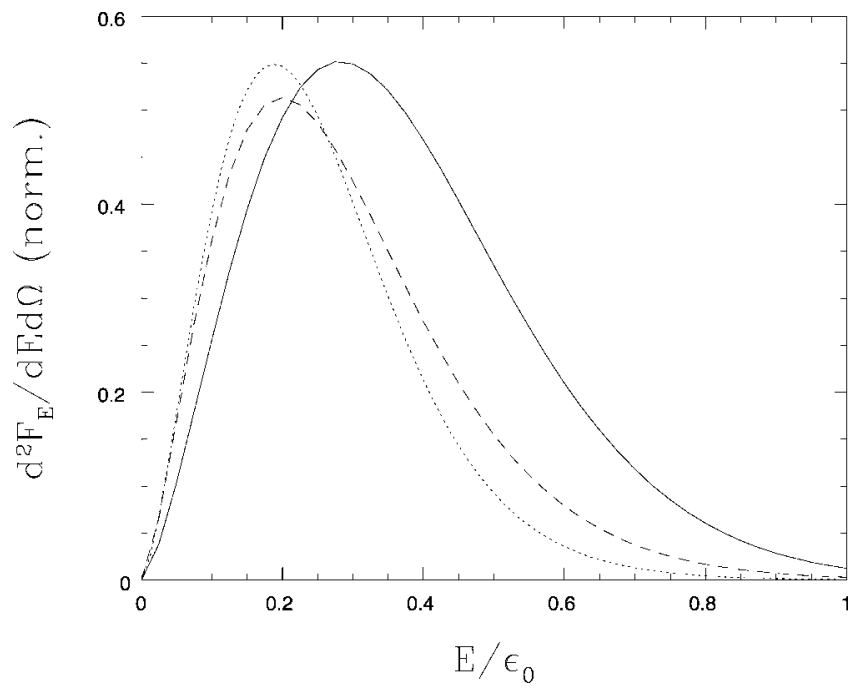

FIG. 3. Same as Fig. 1 but with $\tau H_{0}=0.1$.

$\tau H_{0}=10,1$, and 0.1 , respectively. For an open universe and a flat universe with a nonzero cosmological constant, we choose the values of the density parameters that seem to be observationally favored: $\Omega_{0}=0.3$ and $\Omega_{\Lambda}=0.7$ [8].

For the first model, $\Omega_{0}=1$ and $\Omega_{\Lambda}=0$, one can see that the spectrum has a maximum at

$$
E_{\max }=\left(\frac{3}{2} \tau H_{0}\right)^{2 / 3} \epsilon_{0}=\left(\frac{\tau}{t_{0}}\right)^{2 / 3} \epsilon_{0}=\frac{\epsilon_{0}}{1+z(\tau)}
$$

with $E_{\max }$ in the kinematically allowed region, $E_{\max }<\epsilon_{0}$, as long as $\tau<t_{0}$. The value of the normalized spectrum at the maximum is $\left(d^{2} F_{E} / d E d \Omega\right) /\left(\tilde{n}_{\nu_{i}}\left(t_{0}\right) / 4 \pi\right)=3 / 2 e$ $=0.5518 \ldots$ independent of $\tau H_{0}$. As $\tau H_{0}$ decreases the shape of the spectrum becomes narrower and peaked to lower values of $E$. This reflects the fact that when the lifetime $\tau$ is smaller than the age of the universe $t_{0}$, most neutrinos have already decayed, and the energy of the photons produced in the decay has been redshifted to low values of $E$. The differences in the spectrum for the different cosmological models are easy to understand. Let us compare the critical matter-dominated model with the flat model with nonvanishing $\Lambda$ (the open model falls in between). For $E \rightarrow 0$ the photons are produced at large $z_{0}$, when the Hubble expansion factor for the nonvanishing $\Lambda$ model is smaller than $H(z)$ in the critical matter-dominated model by a factor $\sqrt{1-\Omega_{\Lambda}}$, while the term $\exp -t / \tau$ in Eq. (9) becomes unity for both models. Therefore, the spectrum is higher when $\Lambda>0$ for $E$ small. On the other hand, when $E \rightarrow \epsilon_{0}$, the photons have just been produced, at the same expansion rate $H_{0}$ but with an age for the $\Lambda$ model older than the age for the critical matterdominated case. Consequently, the term $\exp -t / \tau$ makes the spectrum for the critical matter-dominated model higher than for the flat nonzero $\Lambda$ model in the range of large $E$. The position of the maximum in the spectrum when one has a nonvanishing $\Lambda$ is given by 


$$
\begin{aligned}
E_{\max } & =\left(\frac{3}{2} \tau H_{0}\right)^{2 / 3}\left(\frac{2 \Omega_{0}}{1+\sqrt{1+9 \Omega_{\Lambda} \tau^{2} H_{0}^{2}}}\right)^{2 / 3} \epsilon_{0} \\
& <\left(\frac{3}{2} \tau H_{0}\right)^{2 / 3} \epsilon_{0},
\end{aligned}
$$

which is smaller than the value of $E_{\max }$ when $\Lambda=0$.

We have shown that the differences in the shape of the photon spectrum introduced by the cosmological energy density parameters are small. Only a very accurate determination of the photon spectrum by observation could be useful to discriminate among different cosmological models, provided that neutrinos turned out to be unstable with a priori known $\tau H_{0} \sim 1$. On the other hand, if the photon spectrum was well measured and $\tau$ was unknown, the small sensitivity of the spectrum to the energy contents of the universe would allow a fair determination of $\tau$.

\section{UNSTABLE NEUTRINOS WITH A DISTRIBUTION OF MOMENTA}

In this section we shall calculate the spectrum of photons produced by a cosmic background of unstable neutrinos which are not at rest but decay with a statistical distribution of momenta $f_{i}\left(\vec{p}_{i}\right)$. Besides the expansion and geometric effects discussed in Sec. II, the photon spectrum will also include features arising from this momentum distribution.

As always we consider the two-body decay

$$
\nu_{i}\left(\epsilon_{i}, \vec{p}_{i}\right) \rightarrow \nu_{j}\left(\epsilon_{j}, \vec{p}_{j}\right)+\gamma(\epsilon, \vec{p})
$$

where the energy and three momentum of each particle are indicated. A photon is produced with an energy $\epsilon$ at time $t(z)$; at present its energy has been redshifted to $E$ with $\epsilon$ $=(1+z) E$.

The present photon energy flux per solid angle with energy between $E$ and $E+d E$ can be written as

$$
\frac{d^{2} F_{E}}{d \Omega}=\frac{1}{4 \pi} \int \frac{d^{3} \vec{p}}{(2 \pi)^{3}} \frac{1}{(1+z)^{3}} E \delta f_{\gamma}(\vec{p}, z)
$$

where $d^{3} \vec{p} /(2 \pi)^{3} \delta f_{\gamma}(\vec{p}, z)$ is the photon number density, including both photon helicities, produced by neutrinos decaying at redshift between $z$ and $z+d z$; these photons are produced with energy between $\epsilon$ and $\epsilon+d \epsilon$. The origin of the term $1 /(1+z)^{3}$ is the same as when the $\nu_{i}$ 's decay at rest. The increment $\delta f_{\gamma}$ is given by the collision term of the Boltzmann equation [9]

$$
\begin{aligned}
\delta f_{\gamma}(\vec{p}, z)= & \frac{1}{2 \epsilon} \int \frac{d^{3} \vec{p}_{i}}{(2 \pi)^{3} 2 \epsilon_{i}} \int \frac{d^{3} \vec{p}_{j}}{(2 \pi)^{3} 2 \epsilon_{j}} \\
& \times(2 \pi)^{4} \delta^{(4)}\left(p_{i}-p_{j}-p\right)|\mathcal{M}|^{2} \\
& \times\left[f_{i}\left(1-f_{j}\right)\left(1+f_{\gamma}\right)-\left(1-f_{i}\right) f_{j} f_{\gamma}\right] \delta t
\end{aligned}
$$

The functions $f_{j}$ and $f_{\gamma}$ are the statistical momentum distributions of the decay products $\nu_{j}$ and $\gamma$, respectively. The averaged square probability amplitude of the decay (21) is given by

$$
|\mathcal{M}|^{2}=16 \pi \frac{m_{i}^{3}}{\Delta m^{2}} \frac{1}{\tau}
$$

For a fixed redshift $z$ (or fixed $\epsilon$ ) the time increment is $\delta t$ $=H(z)^{-1} d E / E$. The inverse process $\gamma \nu_{j} \rightarrow \nu_{i}$ can be neglected when $m_{i} \gg m_{j}$ since at the late times $\left(\tau \sim H_{0}^{-1}\right)$, we are interested in the photon energy that is about $10^{-4} \mathrm{eV} \sim 3$ $\mathrm{K}$, much smaller than the neutrino mass $m_{i} \sim 0.1 \mathrm{eV}$. In order to obtain analytic expressions for the final spectrum we will also neglect Pauli blocking and stimulated emission, which are expected to produce small corrections. With these approximations we can write

$$
\begin{aligned}
\frac{d^{2} F_{E}}{d E d \Omega}= & \frac{1}{4 \pi} \int \frac{d^{3} \vec{p}}{(2 \pi)^{3} 2 \epsilon} \int \frac{d^{3} \vec{p}_{i}}{(2 \pi)^{3} 2 \epsilon_{i}} \int \frac{d^{3} \vec{p}_{j}}{(2 \pi)^{3} 2 \epsilon_{j}} \\
& \times(2 \pi)^{4} \delta^{(4)}\left(p_{i}-p_{j}-p\right)|\mathcal{M}|^{2} \frac{1}{H(z)} \frac{f_{i}}{(1+z)^{3}} .
\end{aligned}
$$

We shall now relate $f_{i}$ at time $t(z)$ with the distribution function $\tilde{f}_{i}$ that the neutrinos $\nu_{i}$ would have at present if they had not decayed. The evolution of $f_{i}$ is governed by the Boltzmann equation

$$
\begin{aligned}
\frac{d f_{i}}{d t}= & -\frac{1}{2 \epsilon_{i}} \int \frac{d^{3} \vec{p}_{j}}{(2 \pi)^{3} 2 \epsilon_{j}} \int \frac{d^{3} \vec{p}}{(2 \pi)^{3} 2 \epsilon} \\
& \times(2 \pi)^{4} \delta^{(4)}\left(p_{i}-p_{j}-p\right)|\mathcal{M}|^{2} \\
& \times\left[f_{i}\left(1-f_{j}\right)\left(1+f_{\gamma}\right)-\left(1-f_{i}\right) f_{j} f_{\gamma}\right] .
\end{aligned}
$$

Neglecting Pauli blocking, Bose stimulation, and the inverse process, and using Eq. (24) we get the differential equation

$$
\frac{d f_{i}\left(\vec{p}_{i}(t), t\right)}{d t}=-\frac{1}{\tau} \frac{m_{i}}{\epsilon_{i}} f_{i}\left(\vec{p}_{i}(t), t\right),
$$

which can easily be solved to obtain

$$
f_{i}\left(\vec{p}_{i}(t), t\right)=f_{i}\left(\vec{p}_{i}\left(t_{p}\right), t_{p}\right) \exp -\int_{t_{p}}^{t} \frac{d t^{\prime}}{\tau} \frac{m_{i}}{\epsilon_{i}\left(t^{\prime}\right)}
$$

where we choose the arbitrary constant time $t_{p}$ to be $t_{p} \ll \tau$. Including the $(1+z)^{-3}$ that appears in Eq. (25) we can write 


$$
\begin{aligned}
\frac{f_{i}\left(\vec{p}_{i}(t), t\right)}{(1+z)^{3}} d^{3} \vec{p}_{i}(t)= & \frac{f_{i}\left(\vec{p}_{i}\left(t_{p}\right), t_{p}\right)}{\left[1+z\left(t_{p}\right)\right]^{3}} d^{3} \vec{p}_{i}\left(t_{p}\right) \\
& \times \exp -\int_{t_{p}} \frac{d t^{\prime}}{\tau} \frac{m_{i}}{\epsilon_{i}\left(t^{\prime}\right)} \\
= & \widetilde{f}_{i}\left(\vec{q}, t_{0}\right) d^{3} \vec{q} \exp -\int_{t_{p}}^{t} \frac{d t^{\prime}}{\tau} \frac{m_{i}}{\epsilon_{i}\left(t^{\prime}\right)},
\end{aligned}
$$

where $\vec{q} \equiv \vec{p}_{i}\left(t_{p}\right) /\left[1+z\left(t_{p}\right)\right]=\vec{p}_{i}(t) /(1+z)$ is the neutrino momentum at $t_{0}$. The distribution function $\widetilde{f}_{i}\left(\vec{q}, t_{0}\right)$ would be the neutrino distribution function at present if the $\nu_{i}$ 's were stable. In Appendix A we derive its expression.

Making use of Eqs. (24), (25), and (29) we get

$$
\begin{aligned}
\frac{d^{2} F_{E}}{d E d \Omega}= & \frac{1}{4 \pi} \int \frac{d^{3} \vec{p}}{(2 \pi)^{3} 2 \epsilon} \int \frac{d^{3} \vec{q}}{(2 \pi)^{3} 2 \epsilon_{i}} \int \frac{d^{3} \vec{p}_{j}}{(2 \pi)^{3} 2 \epsilon_{j}} \\
& \times(2 \pi)^{4} \delta^{(3)}\left(\vec{p}_{i}-\vec{p}_{j}-\vec{p}\right) \delta\left(\epsilon_{i}-\epsilon_{j}-\epsilon\right) \\
& \times 16 \pi \frac{m_{i}^{3}}{\Delta m^{2}} \frac{1}{\tau} \frac{1}{H(z)} \widetilde{f}_{i}\left(\vec{q}, t_{0}\right) \exp -\int_{t_{p}}^{t} \frac{d t^{\prime}}{\tau} \frac{m_{i}}{\epsilon_{i}\left(t^{\prime}\right)}
\end{aligned}
$$

with

$$
\begin{aligned}
\epsilon_{i, j} & =\sqrt{m_{i, j}^{2}+\vec{p}_{i, j}^{2}}, \\
\vec{p}_{i} & =(1+z) \vec{q}, \\
\epsilon & =|\vec{p}|=(1+z) E .
\end{aligned}
$$

The momentum $\vec{p}_{j}$ can be integrated out using $\delta^{(3)}\left(\vec{p}_{i}-\vec{p}_{j}\right.$ $-\vec{p})$. The lower limit of the integral in the exponential can be set to $0, t_{p}=0$. Assuming isotropy for $\tilde{f}_{i}$ and using Eq. (33) we can finally write the following expression for the photon spectrum produced by the decaying neutrinos

$$
\frac{d^{2} F_{E}}{d E d \Omega}=\int_{0}^{t_{0}} d t I(z(t))=\int_{0}^{\infty} \frac{d z}{1+z} \frac{1}{H(z)} I(z),
$$

where $I(z(t))$ is the energy flux per unit photon energy and the solid angle produced between $t$ and $t+d t$ and is given by

$$
\begin{aligned}
I(z) \equiv & \frac{1}{4 \pi} \int \frac{d^{3} \vec{q}}{(2 \pi)^{3}} \tilde{f}_{i}(\vec{q}) \frac{1}{\tau} \frac{m_{i}}{\epsilon_{i}} \exp -\frac{\mathcal{T}(y, z)}{\tau}(1+z) E \\
& \times\left(\frac{1+z}{1+z_{0}}\right)^{2} \delta\left((1+z) E-\frac{\Delta m^{2} / 2}{\epsilon_{i}-\left|\vec{p}_{i}\right| \cos \theta}\right) .
\end{aligned}
$$

The redshift $z_{0}$ has been defined in Eq. (3). The angular variable $\theta$ is the angle between the momentum of the decaying neutrino and the momentum of the emitted photon, and

$$
y \equiv \frac{|\vec{q}|}{m_{i}}
$$

We have defined the "kinematically contracted expansion time,"

$$
\begin{aligned}
\mathcal{T}(y, z) & \equiv \int_{0}^{t(z)} d t \frac{m_{i}}{\epsilon_{i}(z(t))} \\
& =\int_{z}^{\infty} \frac{d z^{\prime}}{1+z^{\prime}} \frac{1}{H\left(z^{\prime}\right)} \frac{1}{\sqrt{1+y^{2}\left(1+z^{\prime}\right)^{2}}},
\end{aligned}
$$

which reduces to the ordinary expansion time only if $y=0$, $\mathcal{T}(0, z)=t(z)$. In Appendix B we calculate analytically the function $\mathcal{T}(y, z)$ for the three scenarios defined in Sec. II.

Equation (34) has to be multiplied by a factor 2 if, as is usually assumed, there is a cosmological population of antineutrinos $\bar{\nu}_{i}$ with the same distribution function as the $\nu_{i}$ and the $C P$ symmetry is not broken in the decay.

The physical meaning of the different terms in Eq. (35) is straightforward: there is a sum over all possible momenta with which the neutrinos $\nu_{i}$ can decay, each particular momentum weighed by the decay probability per unit time $\tau^{-1}\left(m_{i} / \epsilon_{i}\right) \widetilde{f}_{i} \exp -\mathcal{T} / \tau$. The time dilation factor $\epsilon_{i} / m_{i}$ that appears multiplying $\tau$, in the factor before the exponential and in the exponent, takes into account the relativistic delay in the decay of moving neutrinos. The term $(1+z)$ accompanying $E$ stems from the cosmic expansion, which redshifts the photon energy. The term $\left[(1+z) /\left(1+z_{0}\right)\right]^{2}$ accounts for the photon phase space. Finally, Dirac's $\delta$ enforces energy conservation. Each photon is Doppler shifted by the motion of the neutrino that emits it. The energy of the emitted photon in the rest frame of the decaying neutrino $\epsilon_{0}$ is blueshifted if $\cos \theta>0$ (emitter $\nu_{i}$ and emitted $\gamma$ moving in the same direction) or redshifted if $\cos \theta<0$ ( $\nu_{i}$ and $\gamma$ moving in opposite directions).

Equations (34) and (35) are the main result of this section. They give the spectrum of photons produced by the decay of a cosmic background of neutrinos. They hold for an arbitrary Robertson-Walker geometry and equation of state of the universe, and for any isotropic, nondegenerate momentum distribution of the neutrino background.

As a consistency check we evaluate Eqs. (34) and (35) when $\widetilde{f}_{i}(\vec{q})=(2 \pi)^{3} \widetilde{n}_{\nu_{i}}\left(t_{0}\right) \delta^{(3)}(\vec{q})$, i.e., all the $\nu_{i}$ are at rest, and we recover Eq. (9).

We can also calculate the total photon flux per solid angle at the present time produced by unstable neutrinos with an arbitrary $\widetilde{f}_{i}(\vec{q})$

$$
\begin{aligned}
\frac{d F_{n}}{d \Omega} & \equiv \int \frac{d E}{E} \frac{d^{2} F_{E}}{d E d \Omega} \\
& =\frac{1}{4 \pi} \int \frac{d^{3} \vec{q}}{(2 \pi)^{3}} \widetilde{f}_{i}(\vec{q})\left(1-\exp -\frac{\mathcal{T}(y, 0)}{\tau}\right) .
\end{aligned}
$$

If $\tau \ll H_{0}^{-1}$ we find 


$$
\frac{d F_{n}}{d \Omega}=\frac{1}{4 \pi} \int \frac{d^{3} \vec{q}}{(2 \pi)^{3}} \widetilde{f}_{i}(\vec{q})=\frac{1}{4 \pi} \tilde{n}_{\nu_{i}}\left(t_{0}\right),
$$

as one should expect, since in this regime all $\nu_{i}$ have already decayed into photons.

Next we shall consider the example in which $\widetilde{f}_{i}$ is given by the momentum distribution of the standard stable neutrino background (see Appendix A)

$$
\widetilde{f}_{i}(\vec{q})=\frac{1}{\exp |\vec{q}| / T_{0}+1}
$$

where $T_{0}$ is the present neutrino temperature and we have set the neutrino chemical potential to zero. We shall study the limit $m_{i} \gg T_{0}$. The motion of the nonrelativistic $\nu_{i}$ will introduce small corrections to the photon spectrum when compared to the spectrum produced by neutrinos decaying at rest. In order to calculate these corrections we shall need the asymptotic expressions of $\mathcal{T}(y, z)$ when $y \equiv|\vec{q}| / m_{i} \rightarrow 0$, which are calculated in Appendix B. In most cases we can write

$$
H_{0} \mathcal{T}(y, z)=H_{0} t(z)-\alpha y^{n}+\cdots
$$

where $\alpha$ is a constant and we neglect terms that decrease faster than $y^{n}$ when $y \rightarrow 0$. Plugging Eqs. (41) and (40) into Eqs. (34) and (35) we obtain

$$
\begin{aligned}
\frac{d^{2} F_{E}}{d E d \Omega}= & \frac{1}{4 \pi} \frac{\tilde{n}_{\nu_{i}}\left(t_{0}\right)}{\tau H\left(z_{0}\right)} \exp -\frac{t\left(z_{0}\right)}{\tau} \\
& \times\left(1+\frac{\left[2-1 /\left(2^{n+1}\right)\right] \zeta(n+3) \Gamma(n+3) \alpha}{3 \zeta(3) \tau H_{0}}\right. \\
& \left.\times\left(\frac{T_{0}}{m_{i}}\right)^{n}+\ldots\right),
\end{aligned}
$$

where $\zeta(x)$ is the Riemann zeta function and $\Gamma(x)$ is the Euler gamma function. For instance, in a universe with $\Omega_{0}$ $\sim 1$ one has $n=3 / 2$ and $\alpha=\Omega_{0}^{-1 / 2} \Gamma(1 / 4)^{2} / 6 \sqrt{\pi}$, see Eqs. (B9), (B22), and (B26). Equation (42) is then

$$
\begin{aligned}
\frac{d^{2} F_{E}}{d E d \Omega}= & \frac{1}{4 \pi} \frac{\tilde{n}_{\nu_{i}}\left(t_{0}\right)}{\tau H\left(z_{0}\right)} \exp -\frac{t\left(z_{0}\right)}{\tau} \\
& \times\left(1+\frac{35(1-1 / 8 \sqrt{2}) \zeta(9 / 2) \Gamma(1 / 4)^{2}}{48 \zeta(3) \Omega_{0}^{1 / 2} \tau H_{0}}\right. \\
& \left.\times\left(\frac{T_{0}}{m_{i}}\right)^{3 / 2}+\ldots\right),
\end{aligned}
$$

with $\zeta(9 / 2)=1.0547 \ldots, \zeta(3)=1.2021 \ldots$, and $\Gamma(1 / 4)$ $=3.6256 \ldots$.

It is also possible to obtain the following asymptotic form of $\mathcal{T}(y, z)$ :

$$
H_{0} \mathcal{T}(y, z)=H_{0} t(z)+\beta y^{n} \ln y+\cdots
$$

with $\beta$ a constant, which renders

$$
\begin{aligned}
\frac{d^{2} F_{E}}{d E d \Omega}= & \frac{1}{4 \pi} \frac{\tilde{n}_{\nu_{i}}\left(t_{0}\right)}{\tau H\left(z_{0}\right)} \exp \\
& -\frac{t\left(z_{0}\right)}{\tau}\left(1+\frac{\left(4-1 / 2^{n}\right) \zeta(n+3) \Gamma(n+3) \beta}{3 \zeta(3) \tau H_{0}}\right. \\
& \left.\times\left(\frac{T_{0}}{m_{i}}\right)^{n} \ln \frac{m_{i}}{T_{0}}+\cdots\right) .
\end{aligned}
$$

For example, in a critical radiation-dominated universe we have to make use of Eq. (45) with $n=2$ and $\beta=1 / 2$, see Eq. (B11),

$$
\begin{aligned}
\frac{d^{2} F_{E}}{d E d \Omega}= & \frac{1}{4 \pi} \frac{\tilde{n}_{\nu_{i}}\left(t_{0}\right)}{\tau H\left(z_{0}\right)} \exp -\frac{t\left(z_{0}\right)}{\tau} \\
& \times\left(1+\frac{15 \zeta(5)}{2 \zeta(3) \tau H_{0}}\left(\frac{T_{0}}{m_{i}}\right)^{2} \ln \frac{m_{i}}{T_{0}}+\cdots\right),
\end{aligned}
$$

with $\zeta(5)=1.0369 \ldots$

In all the above examples the leading correction is independent of $E$ and positive. There are more photons at present, with a given energy $E$, compared to the case when the neutrinos decay at rest. The dominant effect is the time dilation in the decay of the neutrinos included in $\mathcal{T}(y, z) / \tau$. Photons with present energy $E$ are produced at $1+z \sim \epsilon_{0} / E$; there are more neutrinos available at time $t(z)$ if the decay is delayed, and therefore more photons with energy $E$ can be produced. There is a second effect of time delay that goes in the opposite direction, the number of photons produced at $t(z)$ decreases since the decay probability is inversely proportional to the dilation factor, but this second effect is order $\left(T_{0} / m_{i}\right)^{2}$ and therefore subleading.

We notice that, in general, the natural expansion parameter for $\mathcal{T}(y, z)$ is $y^{1 / 2}$ or equivalently $\left(T_{0} / m_{i}\right)^{1 / 2}$ in the final photon spectrum, if we assume the distribution (40). High precision determination of the photon spectrum would require analytic calculation of higher orders in $\left(T_{0} / m_{i}\right)^{1 / 2}$. In addition, the small corrections introduced by Pauli blocking and stimulated emission can be important when compared with the relativistic corrections we have calculated [6]. An accurate determination of the photon spectrum including all these statistical effects can only be performed numerically, which goes beyond the scope of the present paper. We think that the analytic expressions that we have found can be useful for understanding the physics of a late decaying cosmic neutrino background, in spite of the fact that we neglect contributions that should be included in a complete numerical study.

While in the case that the neutrinos decay at rest, the photon flux vanishes when $E>\epsilon_{0}$, and now it is possible to have a photon flux with energy higher than $\epsilon_{0}$, albeit very small, since when a moving neutrino decays it can emit a Doppler blueshifted photon with energy higher than $\epsilon_{0}$. From Eqs. (34), (35), and (40) we obtain 


$$
\frac{d^{2} F_{E}}{d E d \Omega}=\frac{1}{4 \pi} \frac{\tilde{n}_{\nu_{i}}\left(t_{0}\right)}{\tau H_{0}} \frac{1}{3 \zeta(3)} \frac{m_{i}}{T_{0}}\left(\frac{E}{\epsilon_{0}}\right)^{2} \exp -\frac{1}{2} \frac{m_{i}}{T_{0}} \frac{E}{\epsilon_{0}}
$$

valid for $E \gg \epsilon_{0}, m_{i} \gg T_{0}$, and $\tau H_{0}$ not too small (so that the term $\exp -\mathcal{T} / \tau$ does not dominate the fall of the spectrum).

To end this section we would like to point out that the photon spectrum obtained is not a thermal spectrum even if the neutrinos, which act as the photon source, are distributed thermally. The cosmic expansion and the decay kinematics render a photon distribution that is out of equilibrium. Later interaction of the decay products with matter could restore thermal equilibrium. However, we always assume that the decay happens after decoupling of matter and radiation, i.e., $\tau \gg 300000 \mathrm{yr}$, therefore the decay produced photons never attain thermal equilibrium.

\section{CONCLUSIONS}

In this paper we have studied the contribution to the photon background of a cosmological density of decaying neutrinos. We first have adopted the assumption that neutrinos decay at rest and have investigated the fluxes in different cosmological scenarios: (1) a power-law cosmic expansion, (2) an open universe filled only with matter, and finally (3) a flat universe with matter and a nonzero cosmological constant $\Lambda$. We have calculated the exact formulas for these cases, and found the maximum of the spectrum as a function of the photon energy.

The differences among the scenarios are not large. This result has the consequence that, when the observed photon spectrum is used to constrain the neutrino lifetime, the limit does not depend too much on the energy content of the universe.

Second, we have studied the consequences for the photon background when the decaying neutrinos have a momentum distribution. We have worked out the relevant formulas for the scenarios (1), (2), and (3) mentioned earlier. We have also particularized our equations to the standard neutrino background. In all the cases, to compare with the photon spectrum calculated for neutrinos at rest, we have determined the first relativistic correction in our formulas.

In the literature only the case of neutrinos decaying at rest in a critical matter-dominated universe has been treated; this corresponds to Eq. (14). In this paper we have performed a general study of the photon spectrum that will allow us to obtain reliable bounds on electromagnetic neutrino properties using photon background observations. Also, in the case of an eventual positive signal, the spectrum we have calculated would be helpful to obtain an observational determination of the lifetime $\tau$.

A final comment is that we believe that our calculations may be useful when considering scenarios of radiatively decaying particles, for example, the type considered by Sciama [10] that might be the explanation for the observed ionization of intergalactic hydrogen. As another example, our results can be applied to the contribution to the photon background coming from a relic scalar or pseudoscalar particle density decaying into two photons of the type considered in $[11,12]$.
Of course one should introduce the appropriate changes in the equations of our paper.

\section{ACKNOWLEDGMENTS}

This work was partially supported by the CICYT Research Project AEN98-1116 at IFAE/UAB and by the DOE and NASA Grant No. NAG 5-7092 at Fermilab. R.T. is financially supported by the MEC.

\section{APPENDIX A: DISTRIBUTION OF MOMENTA FOR A STABLE NEUTRINO COSMIC BACKGROUND}

In this appendix we shall study the momentum distribution of a relic cosmic background of stable neutrinos with mass $m$. We neglect the neutrino chemical potential setting it to zero although our results could easily be generalized to include a nonvanishing chemical potential.

Neutrinos decouple from the primordial plasma of photons, electrons and baryons at $t_{d} \sim 1 \mathrm{~s}$, when the temperature is $T_{d} \sim 1 \mathrm{MeV}$. Hereafter, they freely stream without any further nongravitational interactions. Particle number conservation gives the following relation between the momentum distribution at time $t>t_{d}$ and the momentum distribution at decoupling time $t_{d}$

$$
f(\vec{k}, t)=f\left(\vec{k}_{d}, t_{d}\right)
$$

with the neutrino momenta related by $\vec{k}=\vec{k}_{d} a_{d} / a$, a being the cosmic expansion factor. Until time $t_{d}$ the distribution function is given by the Fermi-Dirac thermal distribution, therefore,

$$
f(\vec{k}, t)=\frac{1}{\exp E_{d} / T_{d}+1} .
$$

The energy at decoupling $E_{d}=\sqrt{m^{2}+\vec{k}_{d}^{2}}$ can be written as

$$
\frac{E_{d}}{T_{d}}=\sqrt{\frac{m^{2}}{T_{d}^{2}}+\frac{\vec{k}^{2}}{T^{2}}}
$$

where the neutrino temperature $T$ is related to the neutrino temperature at decoupling $T=T_{d} a_{d} / a$. Writing everything together we obtain

$$
f(\vec{k}, t)=\left(1+\exp \sqrt{\frac{m^{2}}{T_{d}^{2}}+\frac{\vec{k}^{2}}{T^{2}}}\right)^{-1} .
$$

This distribution reduces to a thermal distribution only if the neutrinos are extremely relativistic, $T \gg m$, or nonrelativistic, $T \ll m$, from the decoupling time until the present (for nonrelativistic neutrinos one has to redefine the temperature) [3]. The neutrino temperature $T$ is related to the photon temperature by the relation $T=(4 / 11)^{1 / 3} T_{\gamma}$. We are interested in a neutrino mass about $m \sim 0.1 \mathrm{eV}$, hence the neutrinos are extremely relativistic at the decoupling time but nonrelativistic at present because now $T_{0} \sim 1.7 \times 10^{-4} \mathrm{eV}$. Neglecting the term $m / T_{d} \ll 1$ in Eq. (A4) we finally obtain 


$$
f(\vec{k}, t)=\frac{1}{\exp |\vec{k}| / T(t)+1} .
$$

Note that, in general, this is not a thermal distribution since in the Fermi-Dirac distribution one has $E=\sqrt{m^{2}+\vec{k}^{2}}$ which in our case has been replaced by $|\vec{k}|$. Strictly speaking we cannot call the parameter $T$ the neutrino temperature, but we use this term anyway since it does not cause any confusion. We make use of Eq. (A5) in Sec. III to calculate the spectrum of decay-produced photons.

\section{APPENDIX B: CALCULATION OF THE FUNCTION $\mathcal{T}$}

The integral $\mathcal{T}(y, z)$ defined in Eq. (37) can be solved in terms of elementary and special functions. We shall consider separately the three cases introduced in Sec. II.

\section{Power-law cosmic expansion}

When the Hubble function is given by the power law (11) we have to calculate the following integral:

$$
H_{0} \mathcal{T}(y, z)=\int_{1+z \mathbf{V}^{n+1}}^{\infty} \frac{d \mathbf{v}}{\sqrt{1+y^{2} \mathbf{v}^{2}}},
$$

where $n \geqslant 0$. This integral can be related, by means of the change of integration variable $u=y^{2} \mathrm{v}^{2} /\left(1+y^{2} \mathrm{~V}^{2}\right)$, to the incomplete $\beta$ function, which in turn is related to the hypergeometric function $F(\alpha, \beta ; \gamma ; u)={ }_{2} F_{1}(\alpha, \beta ; \gamma ; u)[13]$.

For $n$ not an even integer we obtain

$$
\begin{aligned}
H_{0} \mathcal{T}(y, z)= & \frac{\Gamma(-n / 2) \Gamma[(n+1) / 2]}{2 \sqrt{\pi}} y^{n} \\
& +\frac{1}{n}\left(\frac{\sqrt{1+y^{2}(1+z)^{2}}}{1+z}\right)^{n} \\
& \times F\left(-\frac{n}{2}, \frac{1-n}{2} ; 1-\frac{n}{2} ; \frac{y^{2}(1+z)^{2}}{1+y^{2}(1+z)^{2}}\right) .
\end{aligned}
$$

For $n$ an even integer, $n>0$, the solution can always be given in terms of elementary functions

$$
\begin{aligned}
H_{0} \mathcal{T}(y, z)= & (-1)^{n / 2} \frac{n !}{(n ! !)^{2}} y^{n} \ln \frac{\sqrt{1+y^{2}(1+z)^{2}}+1}{y(1+z)} \\
& +\frac{\sqrt{1+y^{2}(1+z)^{2}}}{(1+z)^{n}}\left(\frac{1}{n}-\frac{n-1}{n(n-2)} y^{2}(1+z)^{2}\right. \\
& \left.+\ldots(-1)^{1+n / 2} \frac{n !}{(n ! !)^{2}} y^{n-2}(1+z)^{n-2}\right) .
\end{aligned}
$$

Both Eqs. (B2) and (B3) can be written in a more compact but less insightful way using the transformation formulas of the hypergeometric function [13]

$$
\begin{aligned}
H_{0} \mathcal{T}(y, z)= & \frac{1}{n+1} \frac{1}{\sqrt{1+y^{2}(1+z)^{2}}} \frac{1}{(1+z)^{n}} \\
& \times F\left(1, \frac{1}{2} ; \frac{n+3}{2} ; \frac{1}{1+y^{2}(1+z)^{2}}\right),
\end{aligned}
$$

which holds for any $n \geqslant 0$.

As particular examples of Eq. (B2), we write $\mathcal{T}(y, z)$ for a curvature and a flat matter-dominated universe, $n=1,3 / 2$, respectively,

$$
\begin{aligned}
\left.H_{0} \mathcal{T}(y, z)\right|_{n=1}= & -y+\frac{\sqrt{1+y^{2}(1+z)^{2}}}{1+z}, \\
\left.H_{0} \mathcal{T}(y, z)\right|_{n=3 / 2}= & -\frac{1}{6 \sqrt{\pi}} \Gamma(1 / 4)^{2} y^{3 / 2}+\frac{2}{3} \frac{\left[1+y^{2}(1+z)^{2}\right]^{3 / 4}}{(1+z)^{3 / 2}} \\
& \times F\left(-\frac{3}{4},-\frac{1}{4} ; \frac{1}{4} ; \frac{y^{2}(1+z)^{2}}{1+y^{2}(1+z)^{2}}\right) \\
= & -\frac{1}{6 \sqrt{\pi}} \Gamma(1 / 4)^{2} y^{3 / 2}+\frac{2}{3} \frac{\sqrt{1+y^{2}(1+z)^{2}}}{(1+z)^{3 / 2}} \\
& +\frac{1}{3} y^{3 / 2} F\left(\arccos \frac{1-y^{2}(1+z)^{2}}{[1+y(1+z)]^{2}}, \frac{1}{\sqrt{2}}\right) .
\end{aligned}
$$

The function $F(\phi, k)$ is the incomplete elliptic integral of the first kind, see Appendix (B2). In the limit $y \rightarrow 0$ the above expressions become

$$
\begin{gathered}
\left.H_{0} \mathcal{T}(y, z)\right|_{n=1}=\left.H_{0} t(z)\right|_{n=1}-y+O\left(y^{2}\right), \\
\left.H_{0} \mathcal{T}(y, z)\right|_{n=3 / 2}=\left.H_{0} t(z)\right|_{n=3 / 2}-\frac{1}{6 \sqrt{\pi}} \Gamma(1 / 4)^{2} y^{3 / 2}+O\left(y^{2}\right),
\end{gathered}
$$

where $\left.t(z)\right|_{n}$ is given by Eq. (12) and $\Gamma(1 / 4)^{2} / 6 \sqrt{\pi}$ $=1.2360 \ldots$.

As an example of Eq. (B3) one has a critical radiationdominated universe, $n=2$,

$$
\begin{aligned}
\left.H_{0} \mathcal{T}(y, z)\right|_{n=2}= & -\frac{y^{2}}{2} \ln \frac{1+\sqrt{1+y^{2}(1+z)^{2}}}{y(1+z)} \\
& +\frac{1}{2} \frac{\sqrt{1+y^{2}(1+z)^{2}}}{(1+z)^{2}} .
\end{aligned}
$$

In the limit $y \rightarrow 0$ we find

$$
\left.H_{0} \mathcal{T}(y, z)\right|_{n=2}=\left.H_{0} t(z)\right|_{n=2}+\frac{1}{2} y^{2} \ln y+O\left(y^{2}\right),
$$

with $\left.t(z)\right|_{n=2}$ given by Eq. (12).

As a final example of the power law, we consider a critical $\Lambda$-dominated universe, $n=0$. Directly integrating Eq. (B1), or using Eq. (B4) (see the representation of elementary functions in terms of a hypergeometric function in [13]), we obtain 


$$
\left.H_{0} \mathcal{T}(y, z)\right|_{n=0}=\ln \frac{1+\sqrt{1+y^{2}(1+z)^{2}}}{y(1+z)} .
$$

When $y \rightarrow 0$ this expression diverges because $t(z)$ diverges for a critical $\Lambda$-dominated universe.

\section{Open universe}

The integral to solve now is

$$
H_{0} \mathcal{T}(y, z)=\int_{1+z}^{\infty} \frac{d \mathrm{v}}{\sqrt{\Omega_{k} \mathrm{v}^{2}+\Omega_{0} \mathrm{v}^{3}}} \frac{1}{\sqrt{1+y^{2} \mathrm{v}^{2}}}
$$

This integral can be written as

$$
\int_{1+z}^{\infty} \frac{d \mathrm{~V}}{\mathrm{~V}^{2}} \frac{1}{\sqrt{P_{3}(\mathrm{v})}}
$$

with $P_{3}(\mathrm{~V})$ a cubic polynomial, which is a particular case of an elliptic integral [14]. By means of the variable change $\operatorname{cn} u=\operatorname{cn}(u, k)=(\mathrm{V}+a-A) /(\mathrm{V}+a+A), \quad$ where $\quad \mathrm{cn}$ $u$ is the Jacobian cosine amplitude $u, a \equiv \Omega_{k} / \Omega_{0}, A^{2} \equiv a^{2}$ $+1 / y^{2}$, and $k^{2} \equiv(A+a) / 2 A$, the elliptic integral (B13) can be written linearly in terms of elementary functions and the three fundamental incomplete elliptic integrals, of the first kind $F$, second kind $E$, and third kind $\Pi[14,15]$ :

$$
\begin{aligned}
H_{0} \mathcal{T}(y, z)= & \frac{y^{1 / 2}}{\Omega_{k}}\left(\Omega_{0}^{2}+\Omega_{k}^{2} y^{2}\right)^{1 / 4}\left(1-\frac{\Omega_{k} y}{\sqrt{\Omega_{0}^{2}+\Omega_{k}^{2} y^{2}}}\right) F(\phi, k)-\frac{y^{1 / 2}}{\Omega_{k}}\left(\Omega_{0}^{2}+\Omega_{k}^{2} y^{2}\right)^{1 / 4} E(\phi, k) \\
& +\frac{1}{4 \Omega_{k}^{2} y^{1 / 2}} \frac{\left(\sqrt{\Omega_{0}^{2}+\Omega_{k}^{2} y^{2}}-\Omega_{k} y\right)^{2}}{\left(\Omega_{0}^{2}+\Omega_{k}^{2} y^{2}\right)^{1 / 4}} \Pi\left(\phi, n^{2}, k\right) \\
& -\frac{\Omega_{0}}{4 \Omega_{k}^{3 / 2}} \ln \left|\frac{\sqrt{\Omega_{k}} \sqrt{1+y^{2}(1+z)^{2}}+\sqrt{\Omega_{k}+\Omega_{0}(1+z)} \mid}{\sqrt{\Omega_{k}} \sqrt{1+y^{2}(1+z)^{2}}-\sqrt{\Omega_{k}+\Omega_{0}(1+z)}}\right| \\
& +\frac{1}{\Omega_{k}} \frac{\left(\sqrt{\Omega_{0}^{2}+\Omega_{k}^{2} y^{2}}+\Omega_{k} y\right) \sqrt{\Omega_{k}+\Omega_{0}(1+z)}}{\sqrt{\Omega_{0}^{2}+\Omega_{k}^{2} y^{2}}+y\left[\Omega_{k}+\Omega_{0}(1+z)\right]} \frac{\sqrt{1+y^{2}(1+z)^{2}}}{1+z} .
\end{aligned}
$$

We use the following definitions of the incomplete elliptic integrals [14]:

$$
\begin{gathered}
F(\phi, k) \equiv \int_{0}^{\phi} \frac{d \theta}{\sqrt{1-k^{2} \sin ^{2} \theta}}, \\
E(\phi, k) \equiv \int_{0}^{\phi} d \theta \sqrt{1-k^{2} \sin ^{2} \theta}, \\
\Pi\left(\phi, n^{2}, k\right) \equiv \int_{0}^{\phi} \frac{d \theta}{\left(1-n^{2} \sin ^{2} \theta\right) \sqrt{1-k^{2} \sin ^{2} \theta}} .
\end{gathered}
$$

The variables $\phi, k$, and $n$ of the three elliptic integrals are related to the physical variables of our problem:

$$
\begin{aligned}
\cos \phi & \equiv \frac{\left[\Omega_{k}+\Omega_{0}(1+z)\right] y-\sqrt{\Omega_{0}^{2}+\Omega_{k}^{2} y^{2}}}{\left[\Omega_{k}+\Omega_{0}(1+z)\right] y+\sqrt{\Omega_{0}^{2}+\Omega_{k}^{2} y^{2}}}, \\
k^{2} & \equiv \frac{1}{2}\left(1+\frac{\Omega_{k} y}{\sqrt{\Omega_{0}^{2}+\Omega_{k}^{2} y^{2}}}\right),
\end{aligned}
$$

$$
n^{2} \equiv \frac{1}{4 \Omega_{k} y} \sqrt{\Omega_{0}^{2}+\Omega_{k}^{2} y^{2}}\left(1+\frac{\Omega_{k} y}{\sqrt{\Omega_{0}^{2}+\Omega_{k}^{2} y^{2}}}\right)^{2} .
$$

When $y \rightarrow 0$ and $\Omega_{0}$ finite Eq. (B15) reduces to [16]

$$
H_{0} \mathcal{T}(y, z)=H_{0} t(z)-\frac{1}{6 \sqrt{\pi}} \Gamma(1 / 4)^{2} \frac{1}{\Omega_{0}^{1 / 2}} y^{3 / 2}+O\left(y^{2}\right),
$$

where $t(z)$ is given by Eq. (16).

For $\Omega_{0} \rightarrow 0$ and $y$ finite Eq. (B15) gives

$$
\begin{aligned}
H_{0} \mathcal{T}(y, z)= & \left.H_{0} \mathcal{T}(y, z)\right|_{n=1} \\
& +\frac{1}{2}\left(\left.H_{0} \mathcal{T}(y, z)\right|_{n=1}-\ln \frac{1+\sqrt{1+y^{2}(1+z)^{2}}}{y(1+z)}\right) \Omega_{0} \\
& +\cdots,
\end{aligned}
$$

where the ellipsis means smaller contributions like $\Omega_{0}^{2}$, $\Omega_{0}^{2} \ln \Omega_{0}$, and so on.

\section{Flat universe with nonzero $\Lambda$}

In the last case that we study we have to cope with the integral 


$$
H_{0} \mathcal{T}(y, z)=\int_{1+z}^{\infty} \frac{d \mathrm{v}}{\sqrt{\Omega_{\Lambda}+\Omega_{0} \mathrm{v}^{3}}} \frac{1}{\sqrt{1+y^{2} \mathrm{v}^{2}}},
$$

which is of the class

$$
\int_{1+z}^{\infty} \frac{d \mathrm{v}}{\sqrt{P_{5}(\mathrm{v})}}
$$

$P_{5}(\mathrm{v})$ being a five degree polynomial. Integrals of this sort can be solved as linear combinations of elementary functions and hyperelliptic integrals [14]. Now there are five fundamental incomplete hyperelliptic integrals, two of the first kind, two of the second kind, and one of the third kind. Since the solution in terms of the hyperelliptic integrals would not be particularly enlightening, we only study two limiting cases of Eq. (B24).

For $y \rightarrow 0$ and $\Omega_{0}$ finite we find

$$
H_{0} \mathcal{T}(y, z)=H_{0} t(z)-\frac{1}{6 \sqrt{\pi}} \Gamma(1 / 4)^{2} \frac{1}{\Omega_{0}^{1 / 2}} y^{3 / 2}+O\left(y^{2}\right),
$$

where $t(z)$ is given by Eq. (18). In the regime $\Omega_{0} \rightarrow 0$ and $y$ finite we obtain

$$
H_{0} \mathcal{T}(y, z)=\left.H_{0} \mathcal{T}(y, z)\right|_{n=0}-\frac{b}{y} \Omega_{0}^{1 / 3}+\cdots
$$

with

$$
b \equiv 1+\frac{F\left(\frac{2}{3}, \frac{4}{3} ; \frac{5}{3} ; \frac{1-\sqrt{2}}{2}\right)}{2^{4 / 3}(1+\sqrt{2})^{2 / 3}}=1.199 \ldots
$$

[1] Y. Fukuda et al., Phys. Rev. Lett. 81, 1562 (1998).

[2] M.T. Ressell and M.S. Turner, Comments. Astrophys. 14, 323 (1990).

[3] E.W. Kolb and M.S. Turner, The Early Universe (AddisonWesley, Redwood City, CA, 1990).

[4] S.D. Biller et al., Phys. Rev. Lett. 80, 2992 (1998).

[5] G.G. Raffelt, Stars as Laboratories for Fundamental Physics (University of Chicago Press, Chicago, 1996); Phys. Rev. Lett. 81, 4020 (1998).

[6] R. Scherrer and M.S. Turner, Astrophys. J. 331, 33 (1988); G.D. Starkman, N. Kaiser, and R.A. Malaney, ibid. 434, 12 (1994); M. Kawasaki, G. Steigman, and H.-S. Kang, Nucl. Phys. B403, 671 (1993); S. Hannestad, Phys. Rev. D 57, 2213 (1998); S. Hannestad, Phys. Rev. Lett. 80, 4621 (1998); A.D. Dolgov, S.H. Hansen, S. Paston, and D.V. Semikoz, Nucl. Phys. B548, 385 (1999).

[7] For a cosmic fluid with equation of state $p=w \rho$, with $w$ constant, and no spatial curvature, one obtains Eq. (11) with $n$ $=3(1+w) / 2$. The relativistic condition $w \leqslant 1$ constrains $n$ to be $n \leqslant 3$.

[8] A.Dekel, D. Burstein, and S.D.M. White, in Critical Dialogues in Cosmology, edited by N. Turok (World Scientific, Singapore, 1997); S. Perlmutter et al., Nature (London) 391, 51
(1998); B.P. Schmidt et al., Astrophys. J. 507, 46 (1998); A.G. Riess et al., Astron. J. 116, 1009 (1998).

[9] J. Bernstein, Kinetic Theory in the Expanding Universe (Cambridge University Press, Cambridge, England, 1988).

[10] D.W. Sciama, Astron. Astrophys. 335, 12 (1998).

[11] E. Massó and R. Toldrà, Phys. Rev. D 52, 1755 (1995); 55, 7967 (1997).

[12] J.A. Grifols, E. Massó, and R. Toldrà, Phys. Rev. Lett. 77, 2372 (1996); J.W. Brockway, E.D. Carlson, and G.G. Raffelt, Phys. Lett. B 383, 439 (1996).

[13] I.S. Gradshteyn and I.M. Ryzhik, Table of Integrals, Series, and Products (Academic, Orlando, FL, 1980).

[14] P.F. Byrd and M.D. Friedman, Handbook of Elliptic Integrals for Engineers and Scientists (Springer-Verlag, New York, 1971).

[15] Formula 361.54 in [14] contains a misprint when $f_{1}$ is defined therein. The argument in the logarithm should read $\ln \left(\sqrt{\alpha^{2}-1} \mathrm{dn} u+\sqrt{k^{2}+k^{\prime 2} \alpha^{2}} \operatorname{sn} u\right) /\left(\sqrt{\alpha^{2}-1} \operatorname{dn} u\right.$ $-\sqrt{k^{2}+k^{\prime 2} \alpha^{2}}$ sn $\left.u\right)$.

[16] Calculation of the asymptotic forms of $\Pi(\phi, k)$ may be a bit tricky for the not initiated. Use of the infinite series expansion 436.01 in [14] is quite helpful. 